\title{
Treatment of resistant acral vitiligo with fractional Er:YAG laser
}

\author{
Muhsin Al-Dhalimi, ${ }^{*}{ }^{*}$ Haider Al-Sabak, and Aula Hussain ${ }^{b}$
}

\author{
aDepartment of Dermatology, Faculty of Medicine, University of Kufa, Iraq. \\ bDepartment of Dermatology, Karbala Health Directorate, Ministry of Health, Iraq. \\ *Correspondence to Muhsin Al-Dhalimi (email: muhsin.aldhalimi@uokufa.edu.iq) \\ (Submitted: 12 July 2018 - Revised version received: 08 September 2018 - Accepted: 15 September 2018 - Published online: 26 December 2018)
}

\begin{abstract}
Objectives To detect the results of treatment of refractory acral vitiligo by combination of fractional erbium-YAG (Er:YAG) $2940 \mathrm{~nm}$ laser and narrow band-ultraviolet B (NB-UVB) in comparison with NB-UVB alone.

Methods Thirty four patients who had generalized, stable (6 months or more) with resistant acral vitiligo were enrolled. The predominantly affected limbs, lower or upper, were treated with both laser and NB-UVB, while the less predominantly affected limbs were treated with NB-UVB alone. During the treatment period, the patients were seen every 4 weeks regularly.

Results The response rate in areas treated with both fractional Er:YAG laser and NB-UVB was as follows: Four patients (13.3\%) had good response, six patients (20\%) had moderate response and seven patients (23.3\%) had poor response and 13 patients (43.3\%) had no response. The statistical analysis showed a significant response $(P$-value $<0.001)$.

Conclusion Combination therapy is safe and more effective in the treatment of acral vitiligo than NB-UVB monotherapy.

Keywords acral vitiligo, erbium-YAG laser, NB-UVB
\end{abstract}

\section{Introduction}

Vitiligo is an autoimmune, acquired, multifactorial, depigmenting disorder characterized by the appearance of welldemarcated white macules or patches in the skin, due to progressive loss of functional melanocytes in the epidermis. ${ }^{1}$ It has a great cosmetic and psychological effects on the life of affected patients. ${ }^{2}$ Among many clinical types and variants, acral vitiligo is characterized by a high resistance to many treatment modalities. This is probably due to lower hair follicle density, melanocyte density, Langerhans cell density and epidermal MHCII expression. ${ }^{3}$

Narrow band-ultraviolet B (NB-UVB) phototherapy is a very important method in treating vitiligo but the treatment course usually takes long duration maybe 1 year. Skin ablation by erbium-YAG (Er:YAG) laser combined with NB-UVB phototherapy and 5-FU has been tried in the treatment of nonsegmental vitiligo. ${ }^{4}$ It is found to be safe and well-tolerated technique that improves the result of short-term NB-UVB therapy and is anticipated to increase patient compliance. The Er:YAG laser emits light at $2940 \mathrm{~nm}$, with the water affinity being nearly 15 times greater than that of the $\mathrm{CO}_{2}$ laser. This allows the Er:YAG laser to vaporize tissue more efficient, leaving only a small residue of coagulated tissue. As a consequence of the limited photothermal effects, hemostasis and collagen contraction are significantly reduced with Er:YAG lasers.

Accordingly, we investigated the results of treatment of refractory acral vitiligo by combination of fractional Er:YAG $2940 \mathrm{~nm}$ laser and NB-UVB in comparison with NB-UVB alone.

\section{Patients and Methods}

This prospective comparative interventional study was conducted at the Laser Research Unit, College of Medicine, University of Kufa; for the period from November 2014 to December 2015. The ethical approval was obtained from the Scientific Council of Dermatology and Venereology - Iraqi Board for Medical Specializations. Thirty four patients who had generalized, stable (6 months or more) with resistant acral vitiligo were enrolled. Full history and physical examination, assessment of skin phenotypes were done. All patients were types III and IV.

Exclusion criteria included: Pregnancy, lactation, history of cutaneous photosensitivity, eye cataract or skin cancers, localized vitiligo, psychologically unstable patient and those with severe illnesses. Patients who have medical or surgical intervention for vitiligo lesions within last 1 month were also excluded.

The predominantly affected limbs, lower or upper, were treated with both laser and NB-UVB, while the less predominantly affected limbs were treated with NB-UVB alone. Fractional Er:YAG laser 2940 nm (Quanta system DNA laser technology - MATISSE) was used. The parameters of the laser device applied in this study were as follows: Flat tip, fractional, pulse duration $0.3 \mathrm{~ms}$, frequency $2 \mathrm{~Hz}$, and the energy $0.3 \mathrm{~J}$, one pass. Three sessions of fractional Er:YAG laser were performed at 1 month interval. No local anesthesia was needed during laser therapy. Er:YAG laser probe was held perpendicularly on acral part (hands or feet) with one pass of three pulses stumping distributed throughout the lesion.

Full body NB-UVB cabinet (Waldmann, Germany) was used. The treatment started 5 days after each laser session, two times per week on non-consecutive days for 24 sessions. The initial dose was $\left(0.5 \mathrm{~J} / \mathrm{cm}^{2}\right)$ that modified at each session according to the degree of erythema developed during treatment.

During the treatment period, the patients were seen every 4 weeks regularly; at each visit the response and adverse effects were recorded. The patients were followed up each month for 3 months after the last treatment session to look for any complications or any sign of relapse or further improvement.

All vitiliginous lesions were carefully monitored and repeated evaluations were done before each session and at the end of the treatment period. Patients were monitored for repigmentation; overall and per lesional and development of perifollicular pigmentation. 
The clinical response to therapy was visually scored as the percentage of repigmentation of the vitiligenous lesions and rated as follows:

- Excellent response: If $>75 \%$ repigmentation of the depigmented lesions at the end of therapy.

- Good response: If $>50-75 \%$ repigmentation of the depigmented lesions at the end of therapy.

- Moderate response: If $>25-50 \%$ repigmentation of the depigmented lesions at the end of therapy.

- Poor response: If $\leq 25 \%$ repigmentation of the depigmented lesions at the end of therapy.

- No response: If $0 \%$ repigmentation of the depigmented lesions at the end of therapy.

Side effects such as erythema, pruritus and burning sensation were assessed at every follow-up visit.

\section{Photographic Assessment}

Color photographs for each patient were obtained at baseline, and at the end of 12 weeks treatment period. Consecutive photographic documentation were taken every 4 weeks and at the end of the treatment in a good illumination and at the same site, using Sony-digital, high sensitivity, 9.1 mega pixel, DSC-HX1 still camera.

All the treated patients were assessed blindly by two independent board certified dermatologists by Visual Analogue Scale (VAS) of improvement by scale scoring from 0 to 10 .

\section{Patient Satisfaction}

At the end of the sessions, the patients were asked about their satisfaction. The degree of satisfaction was ranged from 0 to 10 .

\section{Results}

Thirty patients with vitiligo completed the study, four patients were defaulted due to inconvenience of the study protocol with their work. There were 17 females $(56.7 \%)$ and 13 males (43.3\%). Their ages ranged from 18 to 64 years with a mean \pm SD $(32.9 \pm 15.2)$ years. Duration of the disease ranged from 1 to 24 years with a mean \pm SD $(8.7 \pm 5.3)$. Ten patients $(33 \%)$ had positive family history.
In this study, the upper limbs were treated in 26 patients with laser and NB-UVB and the lower limbs were treated with $\mathrm{NB}-\mathrm{UVB}$ alone. In the remaining four patients, the lower limbs were treated with laser and NB-UVB and the upper limbs were treated with NB-UVB alone.

The response rate in areas treated with both fractional Er:Yag laser and NB-UVB was as follows: Four patients (13.3\%) had good response, six patients (20\%) had moderate response and seven patients $(23.3 \%)$ had poor response and 13 patients $(43.3 \%)$ had no response. The statistical analysis showed a significant response $(P$-value $<0.001)$. Among the 17 responding patients $(56.7 \%)$, the upper limbs were treated in 13 of them and the lower limbs were treated in the remaining four patients. No response was noticed after the first session of laser. The patient's first response was started after the second session of laser.

The areas treated with NB-UVB alone showed no response at all (Table 1, Figs. 1 and 2).

There was a significant variation in the mean of VAS of improvement between limbs treated with laser and NB-UVB and limbs treated with NB-UVB alone. The average was higher in limbs with combined therapy (Table 2).

The mean score for patient's satisfaction of limbs treated with combined therapy was $4.6000 \pm 3.11393$ while for limbs treated with NB-UVB alone was $0.2667 \pm 0.44978$, so there was highly significant differences between two limbs in each patient $(P$-value $\leq 0.001)$ (Table 3$)$.

\section{Adverse Effect}

Regarding treatment of NB-UVB, one patient developed mild erythema at some stage during treatment that clear without treatment within 24-48 hours after each session and about half of the patients had transient hyperpigmentation that disappeared spontaneously at the end of follow-up period. No patient treated with laser was developed any localized effects. No patient terminates the treatment course because of side effects. No systemic side effect was reported.

\section{Follow-up}

At the end of 3 months of follow-up period after the last sessions, no patient had recurrence or Koebnerization.

\begin{tabular}{|c|c|c|c|c|}
\hline & & \multicolumn{2}{|c|}{ Groups } & \multirow[b]{2}{*}{ P-value } \\
\hline & & $\begin{array}{l}\text { Limbs treated with } \\
\text { laser and NB-UVB }\end{array}$ & $\begin{array}{l}\text { Limbs treated with } \\
\text { NB-UVB alone }\end{array}$ & \\
\hline \multirow{10}{*}{ Response } & & 13 & 30 & \multirow{12}{*}{$<0.001$} \\
\hline & No response & $43.3 \%$ & $100.0 \%$ & \\
\hline & Poor resnonse & 7 & 0 & \\
\hline & rour response & $23.3 \%$ & $0 \%$ & \\
\hline & & 6 & 0 & \\
\hline & Moderate response & $20.0 \%$ & $0 \%$ & \\
\hline & Gend resnence & 4 & 0 & \\
\hline & Goou response & $13.3 \%$ & $0 \%$ & \\
\hline & Excellent resnonse & 0 & 0 & \\
\hline & Excentml tespontse & $0 \%$ & $0 \%$ & \\
\hline \multirow{2}{*}{ Total } & & 30 & 30 & \\
\hline & & $100.0 \%$ & $100.0 \%$ & \\
\hline
\end{tabular}



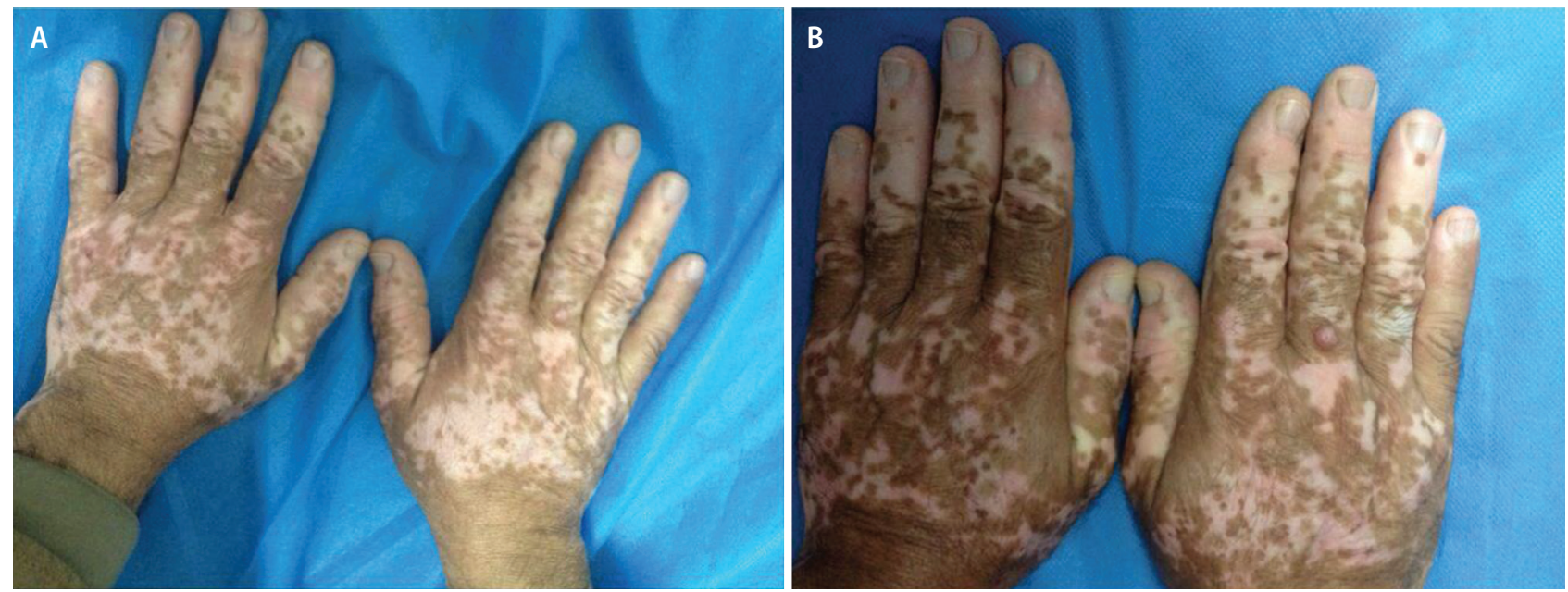

Fig. 1 A 57-year-old male with refractory acral vitiligo, (A) before treatment, (B) after treatment with fractional Er:YAG laser and NB-UVB showing good response.
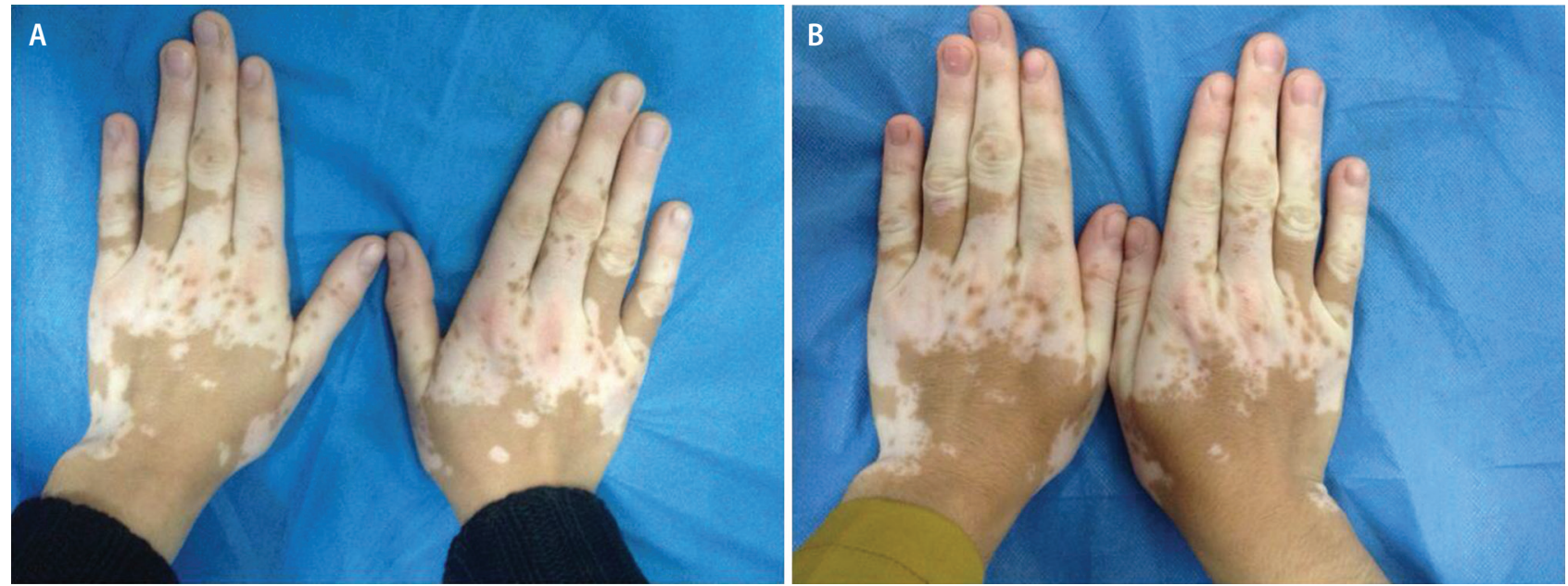

Fig. 2 A 18-year-old female with refractory acral vitiligo, (A) before treatment, (B) after treatment with fractional Er:YAG laser and NB-UVB showing moderate response.

Table 2. Comparison in the VAS of improvement between limbs treated with combined therapy (Er:YAG laser and NB-UVB) and limbs treated with (NB-UVB) alone

\begin{tabular}{clcccc}
\hline & \multicolumn{1}{c}{ Groups } & N & Mean & Std. deviation & P-value \\
\hline \multirow{2}{*}{ Average } & Limbs treated with laser and NB-UVB & 30 & 1.5500 & 1.48179 & 0.011
\end{tabular}

\begin{tabular}{|c|c|c|c|c|c|}
\hline & Groups & $N$ & Mean & Std. deviation & $P$-value \\
\hline \multirow{2}{*}{$\begin{array}{l}\text { Patient } \\
\text { satisfaction }\end{array}$} & Limbs treated with combined therapy & 30 & 4.6000 & 3.11393 & \multirow{2}{*}{$<0.001$} \\
\hline & Limbs treated with NB-UVB alone & 30 & 0.2667 & 0.44978 & \\
\hline
\end{tabular}

\section{Discussion}

Vitiligo is a disfiguring condition that can cause considerable psychological and cosmetic distress to the patient and the family. The lesions on acral areas are considered as difficult to treat. ${ }^{4}$ As conventional treatment of vitiligo on hands and feet results in an unsatisfactory outcome, various ablative treatment options were tried with favorable results.

Combination therapy may produce higher rates of repigmentation compared to traditional monotherapies. So in this 
study, a combination treatment of fractional Er:YAG laser (2940 nm) and NB-UVB was used.

The Er:YAG laser cause vaporization and coagulation. As Er:YAG laser emits light at $2940 \mathrm{~nm}$ with high water affinity, this allows the Er:YAG laser to vaporize tissue more than coagulate, leaving only a small residue of coagulated tissue. So the photothermal effects, hemostasis and collagen contraction are significantly reduced. ${ }^{5}$ Fractionated laser produce microthermal zones. Each microdot of a damage is surrounded by normal, unaffected skin, which acts as a reservoir for healing and enables these microwounds to resolve quickly. ${ }^{4}$ Resurfacing removes the old epidermis that would be replaced by new epidermis formation. This is probably accompanied by production of many growth factors and cytokines like IL6 that cause T cells suppression and TNF, FGF, MMP which stimulate proliferation and migration of residual melanocyte as well producing repigmentation of vitiligo lesion. ${ }^{7}$ This can be regarded as reverse Koebner's phenomenon.

In this work, the patients received three sessions of fractional Er: YAG laser at 1 month interval on the resistant acral lesion, then NB-UVB administrated to the entire body 5 days after each laser session twice a week, for 3 months with gradually increasing dose at each session.

In one study, 5-FU was used in combination with Er:YAG laser in treatment of periungual vitiligo. The response rate was $47.8 \%$ in the treated group while it was $1.1 \%$ in the control group. ${ }^{9}$ This work showed better response rate (50-75\%) in the limbs treated with combination therapy. In another study, NB-UVB was used in addition to 5-FU and one session Er:YAG laser. The results were moderate in $78.1 \%$ of patients as compared with $23.4 \%$ in the mono-therapy group (NB-UVB only). ${ }^{4}$ The results of this study were slightly better than the results of the present work. This is probably due to the addition of NB-UVB to 5-FU and laser therapy.

Potent topical steroids were used in combination with NB-UVB preceded by dermabrasion with of fractional
Er:YAG. The study showed that $50 \%$ of lesions achieved $50 \%$ repigmentation in the dermabrasion side after 1 month, while only $4.2 \%$ with topical steroids and UVB alone. ${ }^{10}$ This study showed better response rate $(50-75 \%)$ in the limbs treated with combination therapy in spite of not using potent topical steroid in our work.

Fractional $\mathrm{CO}_{2}$ laser was used as ablative therapy in other studies instead of Er:YAG laser in difficult to treat areas. ${ }^{7}$ Triple therapy was tried (laser, potent topical steroid, NB-UVB) in refractory vitiligo in two studies. The results showed good repigmentation in $(23.1 \%)$ patients treated with laser, topical steroid and NB-UVB, compared with (3.9\%) in patients without laser treatment. ${ }^{7}$ The results of previous study were better than the present study that showed good response in (13.3\%) the patients. The difference in the results may be attributed to addition of potent topical steroids and more potent dermabrasion effect of fractional $\mathrm{CO}_{2}$ laser in comparison with fractional Er:YAG laser. The other study showed that $44 \%$ of patients achieved over $50 \%$ repigmentation. ${ }^{11}$ The results were comparable with the result of the present work.

In half-body randomized comparative study using $\mathrm{CO}_{2}$ laser treatment followed by NB-UVB, good response was obtained in $10 \%$ of the patients, moderate response in $20 \%$ of patients, poor response in $20 \%$, while in the control group (NB-UVB alone), $20 \%$ of patients showed $25 \%$ response rate. ${ }^{12}$ The sample size in this study was small (10 patients) as compared to this study sample (30 patients), not all treated sites were acral. The results of the present work were also better. In this study, the left and right sides are not compared, because it was cosmetically and psychologically unaccepted for the patients and the patients refused treatment of one side and leaving the other side without laser treatment.

\section{Conflict of Interest}

None.

\section{References}

1. Sandoval-Cruz M, García-Carrasco M, Sánchez-Porras R, Mendoza-Pinto C, Jiménez-Hernández M, Munguía-Realpozo P, et al. Immunopathogenesis of vitiligo. Autoimmun Rev. 2011;10:762-765.

2. Bhatnagar A, Kanwar AJ, Parsad D, De D. Comparison of systemic PUVA and NB-UVB in the treatment of vitiligo: an open prospective study. J Eur Acad Dermatol Venereol. 2007;21:638-642.

3. Esmat SM1, El-Tawdy AM, Hafez GA, Zeid OA, Abdel Halim DM, Saleh MA et al. Acral lesions of vitiligo: why are they resistant to photochemotherapy? J Eur Acad Dermatol Venereol. 2012;26:1097-1104.

4. AnbarTS, WesterhofW, Abdel-Rahman AT, Ewis AA, El-Khayyat MA. Effect of one session of ER:YAG laser ablation plus topical 5Fluorouracil on the outcome of short-term NB-UVB phototherapy in the treatment of non-segmental vitiligo: a left-right comparative study. Photodermatol Photoimmunol Photomed. 2008;24:322-329.

5. Zachary CB. Modulating the Er:YAG laser. Lasers Surg Med. 2000;26:223-226

6. Sami Y. Efficacy of PUVA therapy vs NB-UVB therapy. ARC Dermatol. 2007;143:578-584

7. Vachiramon V, Chaiyabu tr C, Rattanaumpawan P, Kanokrungsee S. Effects of a preceding fractional carbon dioxide laser on the outcome of combined local narrowband ultraviolet B and topical steroids in patients with vitiligo in difficult-to-treat areas. Lasers Surg Med. 2016;48:197-202.

8. Radmanesh M, Sohrabian N, Radmanesh R. Repigmentation of vitiliginous facial area after Q-switched Nd-YAG laser therapy for depigmentation: is it a case of true reverse Koebner phenomenon in vitiligo? Iran J Dermatol. 2013;16:159-161.

9. Anbar T, Westerhof W, Abdel-Rahman A, El-Khayyat M, El-Metwally Y. Treatment of periungual vitiligo with erbium-YAG-laser plus 5-flurouracil: a left to right comparative study. J Cosmet Dermatol. 2006 5:135-139.

10. Bayoumi W, Fontas E, Sillard L, Le Duff F, Ortonne JP, Bahadoran P, et al. Effect of a preceding laser dermabrasion on the outcome of combined therapy with narrowband ultraviolet B and potent topical steroids for treating nonsegmental vitiligo in resistant localizations. Br J Dermatol. 2012;166:208-211.

11. Li L, Wu Y, Li L, Sun Y, Qiu L, Gao XH, et al. Triple combination treatment with fractional CO2 laser plus topical betamethasone solution and narrowband ultraviolet B for refractory vitiligo: a prospective, randomized half-body, comparative study. Dermatol Ther. 2015;28:131-134.

12. Shin J, Lee JS, Hann SK, Oh SH. Combination treatment by $10,600 \mathrm{~nm}$ ablative fractional carbon dioxide laser and narrowband ultraviolet B in refractory non segmental vitiligo: a prospective, randomized half-body comparative study. Br J Dermatol. 2012;166:658-661.

This work is licensed under a Creative Commons Attribution-NonCommercial 3.0 Unported License which allows users to read, copy, distribute and make derivative works for non-commercial purposes from the material, as long as the author of the original work is cited properly. 